\title{
The Han Cultural Factors in the Painted Thangka in Khampa Region and Their Significance in Aesthetic Education
}

\author{
Liu Jun \\ Xi'an University, College of Art, Xi'an, China, 710065
}

\author{
Keywords: Khampa; Thangka; Han Culture; Aesthetic Education
}

Abstract: The painted Thangka art in Khampa region refers to the art style of scroll Buddha paintings popular in Sichuan Ganzi, Tibet Changdu, Yunnan Diqing and other places. In the long process of development over the centuries, this style has calmly maintained the traditional aesthetics of Tibetan Buddhism and absorbed a lot of cultural factors in many Han areas, thus forming a unique Thangka art style. Up to now, these ancient works of art have provided important material information for us to understand the history, politics, religion, culture and other fields of the Tibetan people in the Khampa region and exchanges with the Han culture. At the same time, these Thangka works bear the belief and aesthetic function of the Tibetan people. This paper reveals the catalytic effect of Han culture in the development of Thangka art in the Khampa region, and highlights the aesthetic educational significance of this Thangka art school.

\section{Introduction}

The Tibetan ethnic areas in China are generally divided into three major Tibetan areas, namely, the upper A Li San Wei in the Tibetan history books, the middle Wei Tibetan Si Ru, and the nether Duo Kang Liu Gang. Among them, Duo Kang is divided into two parts, that is, Duo Dui and Duo Mai. Actually, Duokang is Khampa. In the narrow sense of the Khampa region, it refers to Sichuan Ganzi and Aba, Tibet Changdu, Yunnan Diqing and other regions. There are many rivers and mountains in this region, and the people are very strong. It is an extremely important and special part of our Tibetan people. The area not only has temples dating back thousands of years, but also has many famous temples of Tibetan Buddhism Ningma, Gru, Sakya, Kagyu, and Jonang. Besides, the Khampa area is also located at the intersection of the Ancient Tea Horse Road and the South Silk Road. There are many ethnic groups such as Yi, Qiang, Naxi, Hui and Han. At the same time, Confucianism, Taoism, and Han Buddhism in the Central Plains have had direct or indirect effects on the civilization of the Khampa region. These multiculturalism have played an active role in the diversity and richness of the painted Thangka art style in Khampa.

\section{The Origin of Thangka Art}

Regarding the origin of Thangka, there have always been many viewpoints. One viewpoint was proposed by the Italian Tibetan scholar Tu Qi, who believed that Tibet Thangka originated in India. 
Its original form, called pata in India, is a religious painting painted on cotton. This kind of painting is often used in religious ceremonies. This kind of cloth painting is supported by believers as a movable idol. When Buddhism was introduced to Tibet, there was no fixed temple for worship in the early days, so this movable idol was born. Another point of view is that Thangka, which is widely believed in the history of Western art, originated from Newvall paintings in Nepal. The Sanskrit language of this painting is also called pata. Cotton is always used when drawing pata, and the cotton used for painting is specially woven for each picture according to the size of the picture. All Newvall paintings are done using gouache. It is based on the fact that some of the existing early Thangka works mostly belong to the South Asian painting styles such as Nepal. There is also a view that Thangka originated in the 7th century Tubo Zampson, but this view lacks physical evidence. The above statements are different and can only represent their respective opinions. In fact, the true origin of Thangka is complicated by the long time and the annihilation of the Buddha movement in Tibet in history. The cultural relics that can be verified are almost non-existent. Therefore, it is impossible to confirm the origin of Tibetan Thangka. However, according to the "The Sakyamuni Buddha Image. Crystal Treasure Mirror" written by the Fifth Dalai Lama, "Fawang (Songzan Gambo) painted a picture of Bai Lamu with his own nosebleeds. Later, when the Bai Lamu was molded, it was hidden as a sacred object in the belly of Bai Lamu. It can be seen from this historical fact that Thangka appeared in the 7th century AD with the rise of Tibetan Buddhism.

\section{Several Stages of the Development of Thangka in Khampa Region and the Culltural and Artistic Characteristics of Han Culture}

\subsection{Formation Period}

The painted Thangka in Khampa region was formed in the 16th century and flourished in the 17 th century. The famous Thangka painters in this area are mostly Kagyu eminent monk, and they were mainly passed down and popular in the Kagyu temples in the early days. Besides, the Kagyu ceremony was held in the temporary camps, and the term "camp" Tibetan is "ga zi". Therefore, these painters are also collectively referred to as "ga zi school". The early representative of this school was Nan Kazhaxi. He preferred the painting art of Han region in the process of apprenticeship, especially the elegant and delicate performance of Han region meticulous paintings and the strict proportion of the model gave him great inspiration. In order to master these techniques, he has copied a large number of Han region meticulous and freehand works, and combined them with the western "Mian Tang school" that have long been popular in various places in Tibet, invisibly brewing the creative possibilities of integrating the lengths of the families. From the preserved paintings of this period, we can see that the characters in the paintings look like the characters of the Indian era. This is the result of the fact that Nan Kazhaxi still uses the Indian statues and the characteristics of the Mian Tang school to guide the shaping of the characters. The painting and filling of landscapes and various furnishings, flowers, rocks, flowing water, etc. absorb the expression techniques and advantages in the paintings of Han region meticulous paintings, and dyeing maintains the traditional method of thick coating in Tibetan areas.

Although these works combine a variety of styles, they have no sense of clutter, reflecting the ability of the painter to grasp the integration and the overall artistic atmosphere of the picture under the influence of Nan Kazhaxi. In addition, painters of ga zi school in this period also used the scatter perspective method in Han region paintings for the composition, which enhanced the profound effect of the picture. The religious content and the unreachable god Buddha are transformed into lyrics and secular existence. 


\subsection{Heyday}

After the successful exploration of Nan Kazhaxi, a large number of outstanding Thangka painters emerged in the Khampa region. The most representative of them are the tenth Karmapa, Que Ying Duoji (1604-1763), Lang Kajie (1610-1690), and Qu Ji Jiongnai (1700-1774). However, Que Ying Duoji strives for innovation on the success of Nan Kazhaxi's research. He was in a time when the Kagyu school was forced to evacuate from Tibet to Ganzi of Sichuan and Lijiang of Yunnan. He has long been displaced and has no place to live. However, because of this, he has contacted a large number of paintings of the Han region, Naxi and Bai nationalities, fully researching and absorbing the nutrients of these works to perfect and enrich himself. His works such as "Milarepa", "Rohan" and "Sakyamuni" are more integrated into the expression and composition of Han region's meticulous paintings. The color of the picture is magnificent, the landscapes and flowers are very varied, and the characters are ancient and full of vitality. However, the works of Que Ying Duoji's later years are no longer satisfied with the expression of meticulous paintings and move towards freehand painting. The characters he painted have many similarities with the contemporary Han painter Chen Hongshou(1599-1652) . Lang Kajie is the painter of the 17th century Kagyu who is best at depicting miniature Thangka. After studying and drawing on the performance advantages of the green mountains and rivers and the heavy art of the Han region, he applied it to depict the mountains and lakes in the Khampa region. Even many of his works are based on the scenery of the Khampa region, creating a precedent for the scenic Thangka in the Khampa region. The eighth-generation Situ Qu Ji Jiongnai, who was later than him, built the Babang Temple in Dege, as the first regular Thangka learning base in the Khampa region. At the same time, he is also enthusiastic about his creations. Many of his works use the Han region Jinbi landscape technique. The first use of the gold in Chinese paint in the Thangka background, the combination of gold and stone green. The Buddha and the Holy Land symbolized by Thangka is more brilliant and solemn, and the religious connotation of Thangka art is just right. These works are still the model of the Kagyu painter. Since then, the Kagyu school has spread its artistic pursuit of "Chinese localization" to the whole of Tibet with Babang Temple as the center.

\subsection{Modern Period}

In the middle of the 20th century, the Babang Temple monk Tong Laze Weng was the most outstanding member of the group of modern and contemporary painters. In addition to carefully studying the traditional achievements of the old Kagyu, he also absorbed the ancient Thangka techniques that are about to disappear outside the Khampa region and integrated these techniques. His work is made of pure ink or Western watercolor. Besides, he also tried to use the Western realism style as the creative style, and draw on and absorb many elements of interest in the Han culture, such as the magpies, the flower huts, the strange stone and grass, the flowing water and others. In his works, the green mountains and water of the Han region have been thoroughly integrated into the blood of the Kagyu paintings and can be presented in the unique temperament of the Tibetan people. He created a new era of the Kagyu school, and pushed the skills of the Kagyu school to the peak.

\section{The Aesthetic Education Significance of the Han Cultural Factors in the Tangka Art of Kagyu}

\subsection{Artistic Conception of Scene Blending}

The distinctive feature of the Thangka art of Kagyu differs from other genres in its elegant and 
fresh style, which is revealed by the Han Chinese painting aesthetics. It is the idyllic scenery of the wild and detached, the free atmosphere of life and secularization. These characteristics have largely broken through the barriers of Tibetan traditional painting, found a good exit, and embarked on a new and mature path. This kind of innovation makes the Thangka painting different from the religious painting in the traditional sense, which is a serious, cold and quiet atmosphere, and shows a kind of lyrical and free humanism. In these works, the Kagyu painter has skillfully balanced the two domains of religion and secularity.

\subsection{Emphasize Innovation Awareness}

Before the formation of the Kagyu, the Thangka art in the Khampa region basically continued the Tibetan Thangka style. Since Nan Kazhaxi, many typical representatives have courage to explore. They extended their artistic sense to a larger area outside the Tibetan native art tradition. The author once witnessed several traditional line drawing paintings of Tangka in some temples in the Kangpa region. It is obviously a work of learning the paintings of the Han people. According to records, Qu Ji Jiongnai has not only repeatedly studied and translated Chinese Han scriptures, calendars, medicines, paintings and other works in the Central Plains. Moreover, the monks of Babang Temple were asked to go to the Central Plains area to paint the famous mountains and rivers, birds and trees in the Han region into manuscripts and personally hand them to him. These pieces of traditional line drawing Thangka works should be the real works that they learned and explored the Han painting form. Besides, from Nan Kazhaxi to Tong Lazeweng, the entire generation of artists in the Khampa region have been trying to integrate the differences between Tibetan and Han painting. Because these two different characteristics of the painting system have a strong national personality, it is very difficult to achieve a high degree of integration between the two, and the Kagyu painter finally found a match between the two.

\subsection{Innovative Content Is Rich and Broad}

The innovative content of Kagyu is reflected in many aspects, such as the characters in the picture, the processing method of the backlight, the layout of the overall scene, the choice of composition and the arrangement of the dense. These reflect Kagyu's understanding of the Han painting art and the grasp of aesthetic concepts. In particular, the scenery, architecture, and furniture in the background completely reflect the style of the Han region. In terms of perspective arrangement, they abandon South Asian Nepalese cave arrangement method and the average division method of the old Mian Tang school paintings, and adopt the scatter perspective method of Han region meticulous painting, making the picture closer to the green mountains and waters of Han region or Jinbi landscape paintings. In terms of character modeling, they also boldly break through the Nepalese bronze statue style adopted by the traditional Thangka and appropriately use the Han region meticulous and Luohan statues in freehand paintings, which has received very good results.

\section{Conclusion}

In short, the Tibetan community in the Khampa region has long been influenced by religious culture, and the religious culture has a prominent position in the lives of local people. Thangka art not only plays the role of a solemn temple to appease the believers, but also supports the Tibetan people's wedding and funeral, moving to a new home, cultural communication, historical records, cultivation and broadcasting, and communication between the Tibetan and Han nationalities. Therefore, the application of Thangka's works is extremely common, far beyond the scope of 
religion, beyond a certain Tibetan area. In this context, the successful experience of the painters in the Khampa region of Kagyu has not only enriched the expressive ability of Tibetan traditional paintings, but also paved the way for the people of the region to understand the cultural customs of the Han people. By allowing people to appreciate and worship these works, they can also feel the excellence of the two cultures of Han and Tibetan, and ultimately achieve a high degree of spirituality and pleasure and a sense of sublimity. In addition, the successful exploration experience of the Kagyu painters also provided a good model for the Han nationality painters to find new painting styles.

\section{References}

[1] Yu Xiaodong. History of Tibetan Buddhism Painting [M]. Jiangsu: Jiangsu Fine Arts Press. 2006.

[2] He Jing. Zangba Qu Ying Gyatso; A Famous Master in the History of Tibetan Art [J]. Tibetan Human Geography. 2010, 3.

[3] Ge Sangyixi. Tibetan Thangka Art [M]. Beijing: Cultural Relics Press. 2015.

[4] Yang Jiaming. Editor-in-Chief of Mu Ya Dingzeng. Khampa Thangka [M]. Beijing: China Tourism Press. 2010.

[5] Liu Jun. Thangka Art of Tibetan Buddhism [J]. Shenzhou. 2012, 2.

[6] Liu Jun. Research on the Imagery of the Tibetan Buddhism Xianzong Department [J]. Heihe Academic Journal. 2012, 2.

[7] De La Caidan. Thangka Painting Tutorial [M]. Hebei: Hebei Fine Arts Press. 2017. 